\title{
Immune system activation through immunogenic cell death and tumor recruitment of dendritic cells is required for anti-tumor activity of a plant- derived polyphenol rich fraction
}

\author{
Alejandra Gomez-Cadena ${ }^{1 *}$, Amaia Martinez-Usatorre², Claudia Urueña ${ }^{1}$, Karol Prieto ${ }^{1}$, Alfonso Barreto', \\ Pedro Romero², Susana Fiorentino'
}

From 30th Annual Meeting and Associated Programs of the Society for Immunotherapy of Cancer (SITC 2015) National Harbor, MD, USA. 4-8 November 2015

Chemotherapy faces the problem of chemo-resistance in large part of tumors, leading to the appearance of metastasis difficult to eliminate. Over the past decade an improved understanding of the effect of immunogenic chemotherapy on tumor-host interactions has led to the concept of immunogenic cell death (ICD), a type of cell death capable to convert a tumor into an in situ vaccine through the generation of danger signals, dendritic cells activation an finally breaking tolerance, which leads to the destruction of residual tumor cells. Polyphenols are natural compounds from plants, traditionally used for cancer treatment, that exhibit multiple biological activities including anti-oxidation, anti-angiogenesis and proapoptosis, which together may exert potent anti-tumor activity. However, little is known about the mechanism of action of polyphenols. We previously obtained a normalized polyphenol rich fraction from Ceasalpina spinosa (P2Et) that exerted cytotoxic activity on several tumor cell lines. Moreover, P2Et showed anti-tumor activity in the $4 \mathrm{~T} 1$ transplantable model through ICD induction. Vaccination with P2Et-pretreated 4T1 cells yielded long lasting ex vivo IL-2, TNF- $\alpha$, IL-4, IL-5, and IFN $-\gamma$ secreting multifunctional $\mathrm{T}$ cells after specific $4 \mathrm{~T} 1$ cell stimulation. In this work we investigate the activation of the immune system after in vivo P2Et treatment of B16 tumor bearing mice. Analysis of spleen, lymph nodes and tumors shows increase numbers of CD44 high, CD4 and CD8 T cells, as well as NK cells in P2Et

'Grupo de Inmunobiología y Biología Celular. Pontificia Universidad Javeriana, Bogotá - Colombia, Bogota, Colombia

Full list of author information is available at the end of the article treated mice compared to non-treated ones. In addition, in vitro, phagocytosis of P2Et treated tumor cells induce activation of DC, and in vivo P2Et induces recruitment and activation of cross-presenting DCs $\left(\mathrm{CD} 11 \mathrm{c}^{+}, \mathrm{CD} 11 \mathrm{~b}^{+}\right.$, $\mathrm{Ly}_{6 \mathrm{C}^{+}}$or $\mathrm{CD} 11 \mathrm{c}^{+} \mathrm{CD} 8 \alpha^{+}$) in spleen and tumors, which could lead to the effective activation of CD8 T cells favoring a better tumor control. In fact, vaccinated mice treated with P2Et cells are able to effectively control the growth of transplanted tumor. Accordingly, we observed that P2Et anti-tumor activity is highly dependent on an intact immune system, as P2Et anti-tumor activity is lost in RAG $\gamma \mathrm{c}$ KO immunodeficient mice. In summary, P2Et exert its anti-tumor activity through the activation of the endogenous immune system, playing an important role not only in the destruction of the primary tumor but also in controlling metastasis.

\section{Acknowledgements}

Authors thanks the Universidad Javeriana (Grant number: 120110X0401200) and COLCIENCIAS (Grant number: 12011310101103) Bogota, Colombia for financial support and the Colombian Environmental Ministry for allowing the use of genetic resources and derived products (Agreement Number 0454 of 15/05/2013).

\section{Authors' details}

'Grupo de Inmunobiología y Biología Celular. Pontificia Universidad Javeriana, Bogotá - Colombia, Bogota, Colombia. ${ }^{2}$ Ludwig Cancer Research Center, University of Lausanne, Lausanne - Switzerland, Epalinges, Switzerland.

Published: 4 November 2015

doi:10.1186/2051-1426-3-S2-P301

Cite this article as: Gomez-Cadena et al:: Immune system activation through immunogenic cell death and tumor recruitment of dendritic cells is required for anti-tumor activity of a plant-derived polyphenol rich fraction. Journal for ImmunoTherapy of Cancer 2015 3(Suppl 2):P301. 